\title{
Control difuso tipo Mamdani para un brazo-robot con tres grados de libertad
}

Dante Sterpin*

Camilo Giraldo*

David Cañón ${ }^{* * *}$

\section{Resumen}

Los sistemas de razonamiento difuso pretenden simular la capacidad humana de tomar decisiones para actuar en condiciones ambiguas, como, por ejemplo, el hecho de extender el brazo un poco para alcanzar un objeto cercano. El cerebro ejecuta a diario este tipo de acciones sin necesidad de calcular exactamente cuán poco debe extenderse el brazo ni cuán cercano está el objeto. En este artículo se presenta el diseño y los resultados experimentales de un brazo robótico realizado como actividad de semillero de investigación en control difuso. Los resultados muestran que pueden especificarse secuencias de posiciones $(x, y)$ y automáticamente el brazo ejecutará las acciones necesarias para lograrlas.

Palabras clave: brazo-robot, lógica difusa, razonamiento difuso

\section{Abstract}

Fuzzy reasoning systems are intended to simulate the human capacity to make decisions to react in ambiguous situations, such as extending a little the arm to reach something close, a decision that is executed daily by the brain without having to find out exactly how little the arm should extend or how close the object is. This paper presents the design and experimental results of a robotic arm performed as a research seedbed activity in fuzzy control. The results show that a user can specify certain position sequences $(x, y)$, and automatically the robotic arm will execute the necessary actions to achieve them.

Keywords: Fuzzy Logic, Fuzzy Reasoning, Robot-Arm

\footnotetext{
* Ingeniero Electrónico de la Universidad Santo Tomás, Especialista en Docencia Universitaria de la Universidad Militar Nueva Granada y Docente en la Corporación Unificada Nacional de Educación Superior. Contacto: dante_sterpin@cun. edu.co

** Ingeniero Electrónico de la Corporación Unificada Nacional de Educación Superior. Contacto: asinxmr@hotmail.com

*** Ingeniero Electrónico de la Corporación Unificada Nacional de Educación Superior. Contacto: david_a.c.s@hotmail.com
} 


\section{Introducción}

La lógica difusa considera todos los posibles valores de verdad existentes entre los dos valores absolutos de la lógica binaria. Por esta razón, la lógica difusa puede considerarse como una generalización de la lógica binaria. En la teoría clásica de conjuntos, se relacionan los valores de verdad binarios con la pertenencia de un elemento a la característica general de un conjunto, por lo cual es verdadero afirmar que, por ejemplo, una persona de 26 años clasifica como mayor de edad, pero existe cierta dificultad para clasificarla como joven o como adulta.

Dicha dificultad se debe a la ambigüedad lingüística que implica la representación formal de cuestiones tales como la juventud o la adultez. Por tanto, en esta situación resulta apropiado emplear conjuntos difusos, los cuales son funciones matemáticas utilizadas para definir grados de pertenencia, mediante posibles grados de verdad difusos. Teniendo en cuenta la equivalencia entre $\{$ Verdad; Falsedad $\}$ y los valores $\{1$; $0\}$, se dice que los valores de verdad difusos corresponden al rango $[0,0 ; 1,0]$. Así, para el ejemplo de la persona de 26 años, se pueden emplear los conjuntos difusos detallados en la figura $1 \mathrm{y}$ de esta manera puede afirmarse que dicha persona es 0,7 joven y 0,3 adulta.
Esta herramienta permite representar conceptos ambiguos del mundo real mediante la definición de variables lingüísticas, en las cuales se identifica, para cierta cantidad de elementos con alguna característica general, una distribución de conjuntos difusos con el fin de formalizar la concepción de algunos de sus posibles términos. Dichos elementos se agrupan de forma clásica a manera de conjunto universal, denominado universo-de-discurso en el contexto de la lógica difusa. La característica general identifica lo representado por la variable lingüística, mientras que los términos identifican los conjuntos difusos distribuidos en el universo-de-discurso.

Considérese un escenario en el que se tenga que representar las edades humanas. En dicha situación debería agruparse a todas las personas con algún valor de edad en el rango [0; 100] años y se podrían considerar niños, jóvenes, adultos o viejos, como algunas de sus clasificaciones más relevantes. Ese rango de años corresponde al universo-de-discurso, mientras que las cuatro categorías corresponden a los términos cuyos respectivos conjuntos difusos se detallan en la figura 2.

Figura 1. Conjuntos difusos para representar juventud y adultez

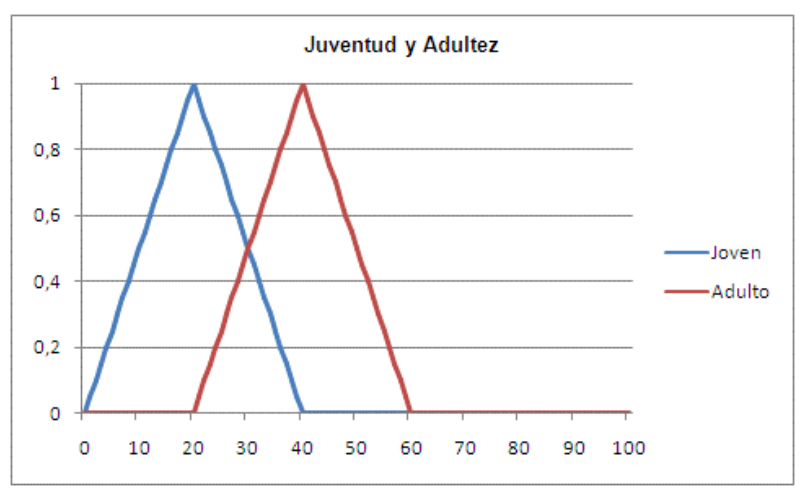

Fuente: elaboración propia 
Figura 2. Conjuntos difusos para representar edades humanas

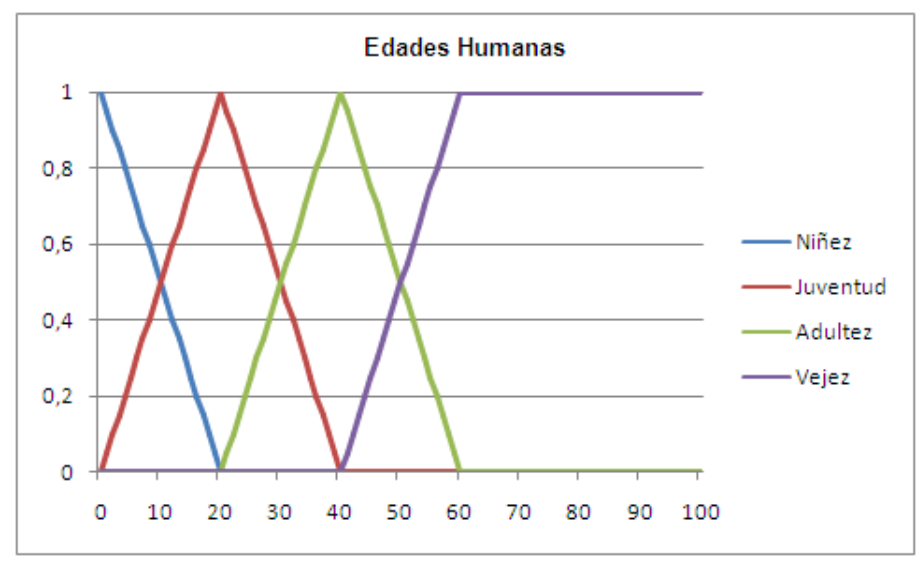

Fuente: elaboración propia

\section{Controlador difuso}

Entre las varias aplicaciones de los sistemas basados en lógica difusa está el control de procesos industriales. El sistema descrito en este artículo controla el posicionamiento de un brazo tipo retroexcavadora, en el cual, cada uno de sus tres grados de libertad está determinado por un servomotor cuyo ángulo varía en el rango [0; 100] grados. Como en esta situación la característica implicada corresponde a la medida de ese ángulo, presente o deseado, los términos apropiados para definir las variables lingüísticas de entrada son: agudo (A), medio-agudo (mA), medio $(\mathrm{m})$, medio-obtuso $(\mathrm{mO})$ y obtuso $(\mathrm{O})$, tal como se indica en las figuras 3 y 4.

Figura 3. Conjuntos difusos para representar el ángulo presente o medido del servomotor

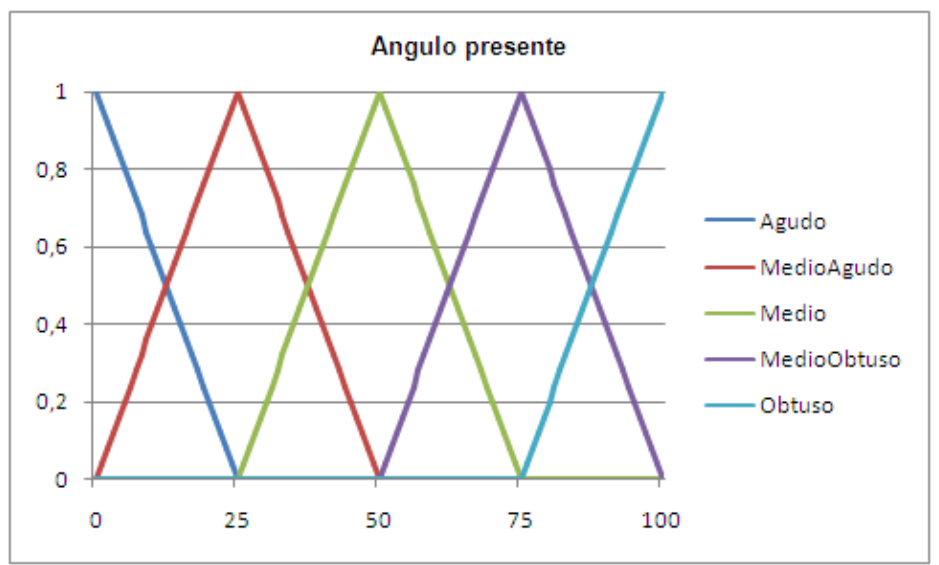

Fuente: elaboración propia 


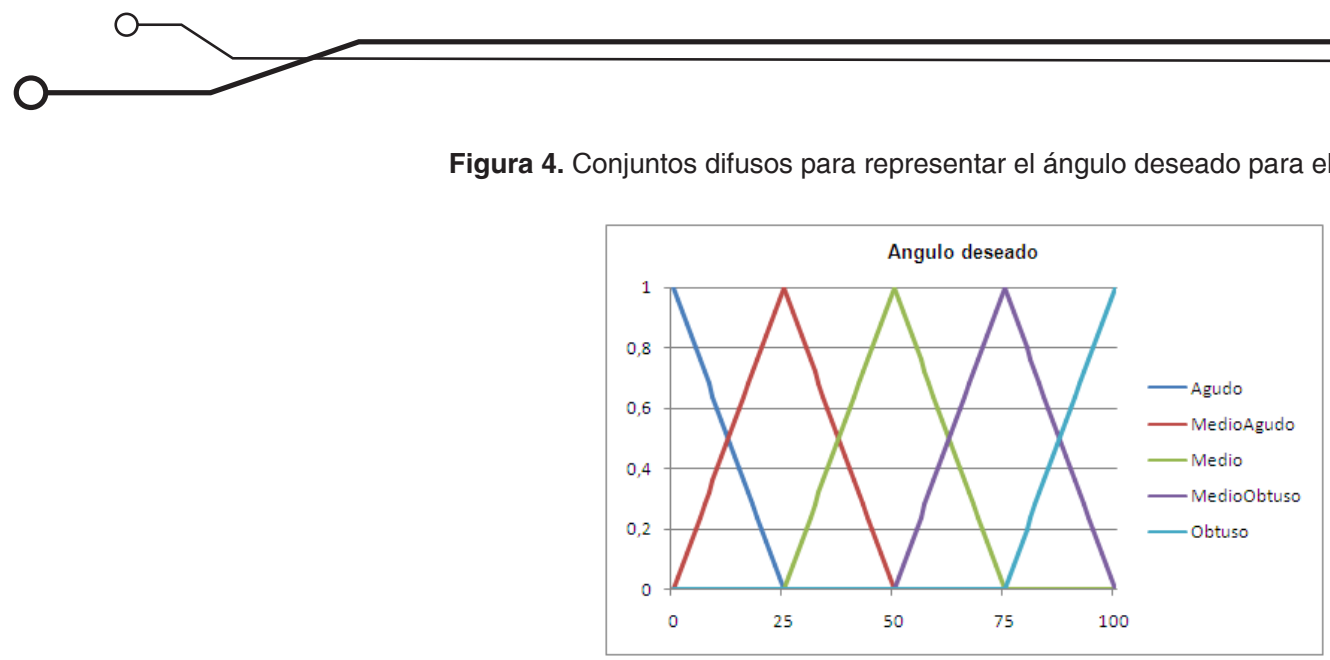

Fuente: elaboración propia

La medida del ángulo presente es obtenida mediante un sensor resistivo tipo reóstato y la medida del ángulo deseado es definida por el usuario vía teclado. De esta manera se tiene el valor real de cada variable lingüística de entrada, con lo que el sistema de control analiza la situación presente y la deseada mediante fuzzyficación. Esto último consiste en calcular los grados de pertenencia correspondientes a los conjuntos difusos de cada variable según su respectivo valor real.

A continuación se muestran las ecuaciones utilizadas para el ángulo presente $\left(\theta_{\mathrm{p}}\right)$, las cuales se aplican de la misma forma para el ángulo deseado $\left(\theta_{\mathrm{d}}\right)$. En las ecuaciones, 1 a la 5 , se detallan las expresiones difusas de los términos lingüísticos, mostrados en las figuras 3 y 4 .

$$
\mu_{A}\left(\theta_{s}\right)=\left\{\begin{array}{ll}
\frac{25-\theta_{s}}{25} & ; \text { para } \theta_{s}<25 \\
0,0 & ; \text { para } \theta_{s} \geq 25
\end{array}\right\}
$$

(Ecuación 1)

$$
\mu_{M A}\left(\theta_{s}\right)=\left\{\begin{array}{lr}
\frac{\theta_{s}}{25} & ; \text { para } \theta_{s} \leq 25 \\
\frac{50-\theta_{s}}{25} & ; \text { para } 25<\theta_{s}<50 \\
0,0 & ; \text { para } \theta_{s} \geq 50
\end{array}\right\}
$$

(Ecuación 2)

$$
\mu_{M}\left(\theta_{s}\right)=\left\{\begin{array}{lr}
0,0 & ; \text { para } \theta_{s} \leq 25 \\
\frac{\theta_{s}-25}{25} & ; \text { para } 25<\theta_{s}<50 \\
\frac{75-\theta_{s}}{25} & ; \text { para } 50 \leq \theta_{s}<75 \\
0,0 & ; \text { para } \theta_{s} \geq 75
\end{array}\right\}
$$

(Ecuación 3)

$$
\mu_{M O}\left(\theta_{s}\right)=\left\{\begin{array}{lr}
0,0 & ; \text { para } \theta_{s} \leq 50 \\
\frac{\theta_{s}-50}{25} & ; \text { para } 50<\theta_{s}<75 \\
\frac{100-\theta_{s}}{25} & ; \text { para } \theta_{s} \geq 75
\end{array}\right\}
$$

(Ecuación 4)

$$
\mu_{o}\left(\theta_{s}\right)=\left\{\begin{array}{ll}
0,0 & ; \text { para } \theta_{s} \leq 75 \\
\frac{\theta_{s}-75}{25} & ; \text { para } \theta_{s}>75
\end{array}\right\}
$$

(Ecuación 5)

Adicionalmente, deben definirse las variables de salida necesarias, según sea el tipo de reacción que permita al ángulo presente acercarse al deseado. Esto se lograría al rotar el eje del servomotor cierta "cuantía", con respecto a su posición actual. Dicha cuantía puede ser: muy negativa $(\mathrm{MN})$, negativa $(\mathrm{N})$, medio negativa $(\mathrm{mN})$, poco pegativa $(\mathrm{PN})$, zero $(\mathrm{Z})$, poco positiva $(\mathrm{PP})$, medio positiva $(\mathrm{mP})$, positiva $(\mathrm{P}), \mathrm{y}$ muy positiva (MP). Al considerar que el rango de posibles 
variaciones es $[-80 ;+80]$, se define una variable lingüística de salida, empleando los conjuntos difusos detallados en la figura 5.

Como cada uno de los tres servomotores tiene la necesidad de rotar cierta cuantía, según la

\section{Razonamiento difuso}

Todo sistema racional debe disponer de cierto conocimiento para poder decidir el cómo actuar en cada posible situación. Como el controlador presentado en este artículo tiene [(101 x 101) x 3] posibles situaciones de ángulo presente $\left(\theta_{p}\right)$ y deseado $\left(\theta_{d}\right)$, sería obsoleto programar explícitamente una reacción apropiada en cada una de ellas, para lo cual puede utilizarse la agrupación mediante los conjuntos difusos de las figuras 3 y 4. Dado lo anterior, se logra representar todo el problema de control con solo [(5 x 5) x 3] posibles combinaciones.

Cada una de dichas combinaciones condiciona una regla difusa de comportamiento, que, formalmente, es una implicación difusa compuesta por una condición y su respectiva reacción, según el tipo de comportamiento esperado para el controlador. Por ejemplo, si en determinada situación el ángulo presente es mA y el ángulo deseado es $\mathrm{mO}$, entonces la reacción necesaria diferencia existente entre sus respectivos ángulos, presente y deseado, entonces se decidió componer el sistema de posicionamiento angular con tres subcontroladores difusos, cada uno con dos variables de entrada y una variable de salida, comentadas previamente.

es aumentar más o menos la posición angular del motor.

Lo anterior podría parecer una forma muy imprecisa de representar el buen funcionamiento del controlador. Sin embargo, el procesamiento numérico de los grados de pertenencia es el garante de la precisión necesaria en la ambigua elasticidad del término medio. Dicho proceso numérico se conoce como inferencia fuzzy y su propósito es calcular un conjunto difuso de decisión-parcial $\mu_{D P}(v)$, en cada regla difusa (R), usando la ecuación 6. Allí, $\mu_{E \rightarrow S}(\mathrm{U} ; \mathrm{v})$ es la relación de implicación entre las entradas y la salida; mientras que $\mu_{S P}(\mathrm{U})$ es el grado de cumplimiento de la situación en la parte condicional de la regla difusa (R), dada la información percibida (U) por el controlador difuso.

$$
\begin{gathered}
\mu_{D P}(v)=\sup _{u \in \mathbf{U}}\left\{\mu_{E \rightarrow S}(\mathbf{U} ; v) ; \mu_{S P}(\mathbf{U})\right\} \\
(\text { Ecuación } 6)
\end{gathered}
$$

Figura 5. Conjuntos difusos para representar la variación angular del servomotor

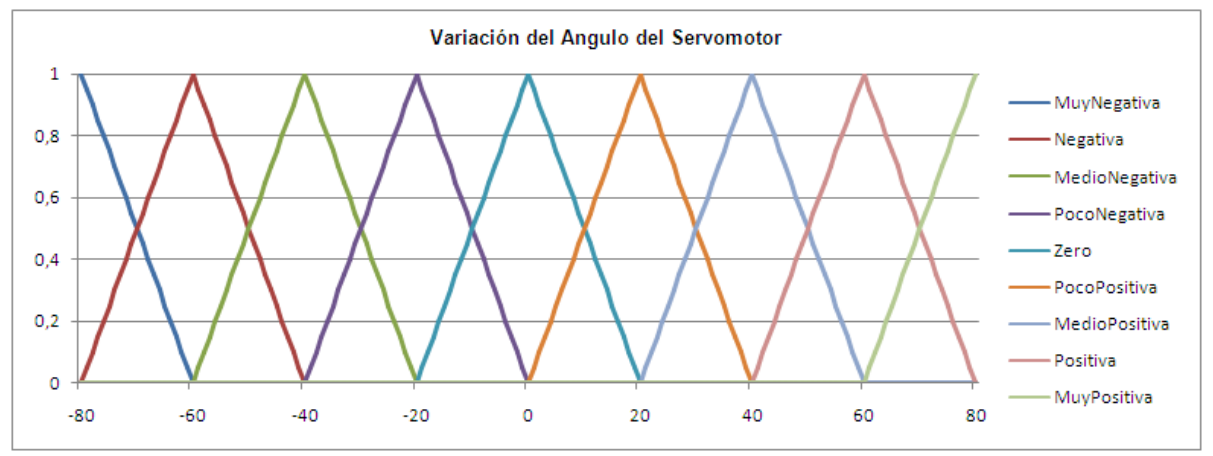

Fuente: elaboración propia 


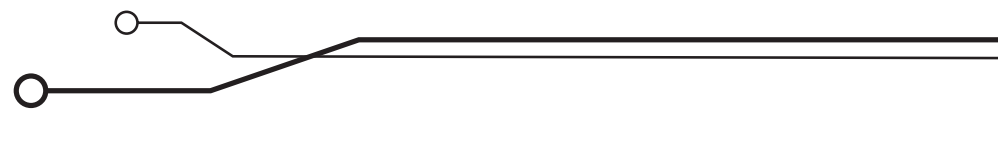

Tras calcular dichos conjuntos difusos, se procede a defuzzyficar una decisión de actuación, es decir, obtener un valor de salida compatible con el mecanismo mediante el cual se modifica el comportamiento del sistema controlado. Para el controlador expuesto en este artículo, la acción consiste en modificar la variable de la cual depende el ángulo de cada servomotor, es decir, el ancho de pulso aplicado, el cual se obtiene mediante la defuzzyficación indicada en la ecuación 7.

$$
v=\frac{\sum_{R}\left[\bar{v} * \mu_{D P}(v)\right]_{R}}{\sum_{R}\left[\mu_{D P}(v)\right]_{R}}
$$

(Ecuación 7)

\section{Conocimiento conductual}

Dado que cada entrada tiene 5 términos, a continuación se especifican las [5 × 5] reglas difusas (R), utilizadas en cada subcontrolador. En cada regla, [u1] simboliza el ángulo presente, [u2] simboliza el ángulo deseado, y [v] simboliza la variación del ángulo.

R1 : Si [u1] es (A) y [u2] es (A), entonces [v] es (Z).

$\mathrm{R} 2$ : Si [u1] es (mA) y [u2] es (A), entonces [v] es (PN).

R3 : Si [u1] es (m) y [u2] es (A), entonces [v] es ( $\mathrm{mN})$.

$\mathrm{R} 4$ : Si [u1] es (mO) y [u2] es (A), entonces [v] es (N).

R5 : Si [u1] es (O) y [u2] es (A), entonces [v] es (MN).

R6 : Si [u1] es (A) y [u2] es (mA), entonces [v] es (PP).

R7 : Si [u1] es (mA) y [u2] es (mA), entonces [v] es (Z)

R8 : Si [u1] es (m) y [u2] es (mA), entonces [v] es (PN).

$\mathrm{R} 9$ : $\mathrm{Si}[\mathrm{u} 1]$ es $(\mathrm{mO})$ y [u2] es $(\mathrm{mA})$, entonces [v] es $(\mathrm{mN})$.

R10 : Si [u1] es (O) y [u2] es (mA), entonces [v] es (N).
Estos dos procedimientos pueden simplificarse mediante la definición de dos matrices, una con los valores de estimulación en cada regla y la otra con los valores de actuación necesaria en cada regla. La primera matriz se calcula escogiendo el mínimo grado de pertenencia entre los calculados para cada combinación de términos en cada posible situación. La segunda matriz se obtiene especificando el valor en el cual está centrado el conjunto difuso del respectivo término de salida definido en cada regla difusa. En la sección donde se aborda la toma de decisión -para la actuación del controlador difuso-, se presentará explícitamente un ejemplo numérico para el controlador presentado en este artículo.

R11 : Si [u1] es (A) y [u2] es (m), entonces [v] es (mP). R12 : Si [u1] es (mA) y [u2] es (m), entonces [v] es (PP).

R13 : Si [u1] es (m) y [u2] es (m), entonces [v] es (Z).

R14 : Si [u1] es (mO) y [u2] es (m), entonces [v] es (PN).

R15 : Si [u1] es (O) y [u2] es (m), entonces [v] es ( $\mathrm{mN})$. $\mathrm{R} 16$ : Si [u1] es (A) y [u2] es (mO), entonces [v] es (P). R17 : Si [u1] es (mA) y [u2] es ( $\mathrm{mO})$, entonces [v] es $(\mathrm{mP})$.

R18 : Si [u1] es (m) y [u2] es (mO), entonces [v] es (PP).

R19 : Si [u1] es (mO) y [u2] es (mO), entonces [v] es (Z).

R20 : Si [u1] es (O) y [u2] es (mO), entonces [v] es $(\mathrm{PN})$.

R21 : Si [u1] es (A) y [u2] es (O), entonces [v] es (MP).

R22 : Si [u1] es (mA) y [u2] es (O), entonces [v] es (P). 
R23 : Si [u1] es (m) y [u2] es (O), entonces [v] es (mP).

R24 : Si [u1] es (mO) y [u2] es $(\mathrm{O})$, entonces [v] es (PP).

R25 : Si [u1] es (O) y [u2] es (O), entonces [v] es (Z).
Tras considerar los conjuntos de la figura 3 y el término de salida detallado en cada regla $(R)$, en la tabla 1 se establecen los valores-centro $(\overline{\mathcal{V}})$ que se emplean en la ecuación 7 para calcular la respuesta del controlador.

Tabla 1. Matriz de centros según el término de salida en cada regla difusa.

\begin{tabular}{|c|c|c|c|c|c|}
\hline $\mathrm{A}$ & 0 & -20 & -40 & -60 & -80 \\
\hline $\mathrm{mA}$ & 20 & 0 & -20 & -40 & -60 \\
\hline $\mathrm{M}$ & 40 & 20 & 0 & -20 & -40 \\
\hline $\mathrm{mO}$ & 60 & 40 & 20 & 0 & -20 \\
\hline $\mathrm{O}$ & 80 & 60 & 40 & 20 & 0 \\
\hline$u 2 / u 1$ & $\mathrm{~A}$ & $\mathrm{~mA}$ & $\mathrm{~m}$ & $\mathrm{mO}$ & $\mathrm{O}$ \\
\hline
\end{tabular}

Fuente: elaboración propia

\section{Decisión de actuación}

Para detallar un ejemplo sobre cómo el controlador difuso toma una decisión de actuación, se asumirá una posible situación asignando estos valores para las entradas: $\{\mathrm{u} 1=29 ; \mathrm{u} 2=64\}, \mathrm{Al}$ fuzzyficarlos se obtienen los grados de pertenencia con respecto a cada término en cada variable de entrada. En la tabla 2 se detallan dichos valores y además se muestran los valores de estimulación en cada regla escogiendo el mínimo valor entre los respectivos grados de pertenencia en u1 y u2.

Tabla 2. Ejemplo de la matriz de estimulación para u1 = 29 y u2 $=64$

\begin{tabular}{|c|c|c|c|c|c|}
\hline 0,0 & 0,0 & 0,0 & 0,0 & 0,0 & 0,0 \\
\hline 0,0 & 0,0 & 0,0 & 0,0 & 0,0 & 0,0 \\
\hline 0,44 & 0,0 & 0,44 & 0,16 & 0,0 & 0,0 \\
\hline 0,56 & 0,0 & 0,56 & 0,16 & 0,0 & 0,0 \\
\hline 0,0 & 0,0 & 0,0 & 0,0 & 0,0 & 0,0 \\
\hline$u 2 / u 1$ & 0,0 & 0,84 & 0,16 & 0,0 & 0,0 \\
\hline
\end{tabular}

Fuente: elaboración propia

Aplicando la ecuación 7 a las tablas presentadas arriba, se obtiene una salida de 23,76. Dicho valor es la decisión de actuación, con cuya ejecución se espera poder aumentar el ángulo presente desde $29^{\circ}$ hasta $(29+23,76)^{\circ}$, procurando así acercarse a los deseados $64^{\circ}$. El efecto real depende del movimiento realizado por el respectivo servomotor y el nuevo ángulo presente se conoce mediante el respectivo sensor de realimentación. 


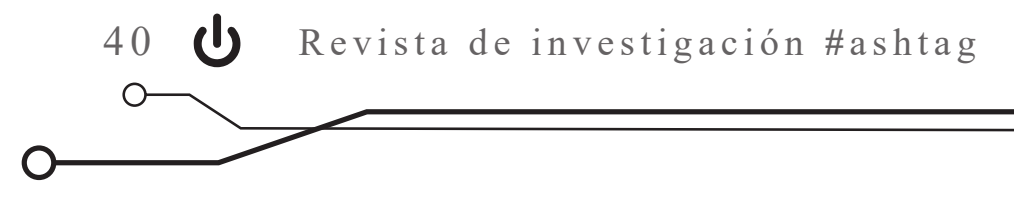

Este procedimiento corresponde a una iteración para un sólo grado de libertad en el brazo, por lo tanto deben ocurrir más iteraciones hasta cuando u1 se iguale a u2, en cada grado de libertad, con lo cual el brazo alcanza cierta postura necesaria a partir de la postura en la que se encuentre inicialmente.

\section{Mapeo entre los ángulos del brazo y su alcance en 2D}

El sistema descrito anteriormente es capaz de posicionar el brazo siempre que conozca los ángulos deseados en cada grado de libertad. Sin embargo, desde el punto de vista del usuario, el brazo debe llegar a cierta coordenada $(x, y)$ desde cualquier otra posición en un espacio 2D. Por lo tanto, el hecho de representar este requerimiento mediante coordenadas es mucho más sencillo en comparación con definir el valor necesario en cada grado de libertad.
De esta manera debe disponerse de un mapeo capaz de indicarle al brazo-robot los valores de ángulos deseados para alcanzar cierto punto $(x$, $y$ ), en una matriz con [22 $\times 26]$ posibles posiciones 2D. La medida de cada posición se especificó en [2 $\times 2] \mathrm{cm}$. para lograr abarcar el alcance total del brazo; su estructura física se muestra en la figura 6, y su espacio de posiciones accesibles se muestra en la figura 7.

Figura 6. Estructura física del brazo utilizado

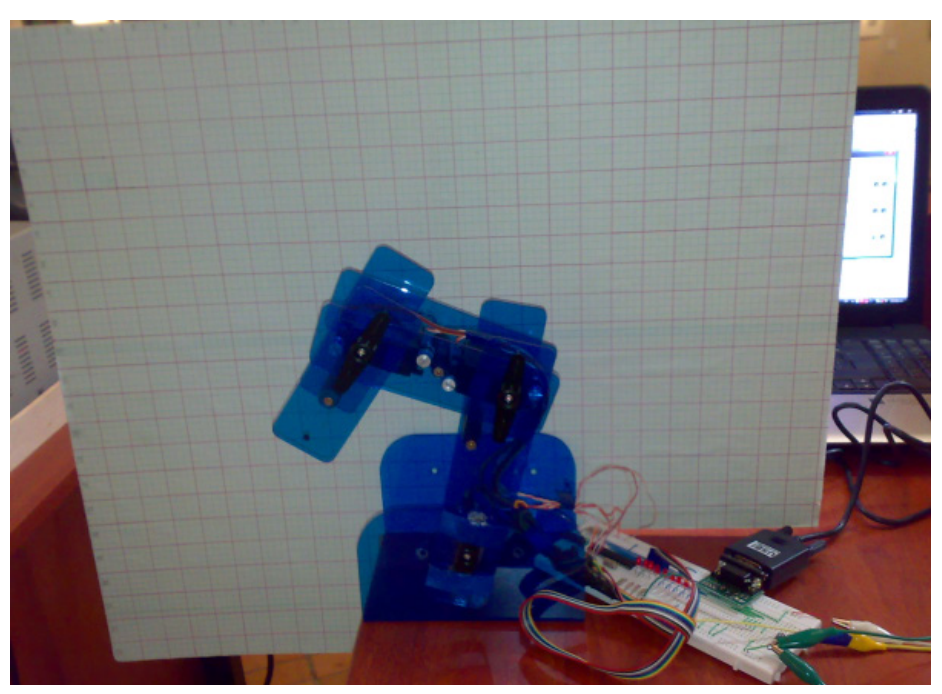

Fuente: elaboración propia

Para obtener la matriz de posiciones 2D realmente accesibles por el brazo, se especificaron 11 valores por cada servomotor: $\{0,10,20,30,40$, $50,60,70,80,90,100\}$ y se combinaron entre sí.
En cada una de las combinaciones se midió la coordenada alcanzada por el brazo y en la posición alcanzada mediante varias combinaciones se prefirió la que posicionaba mejor al brazo. 
Figura 7. Matriz de posiciones realmente accesibles por el brazo, en 2D

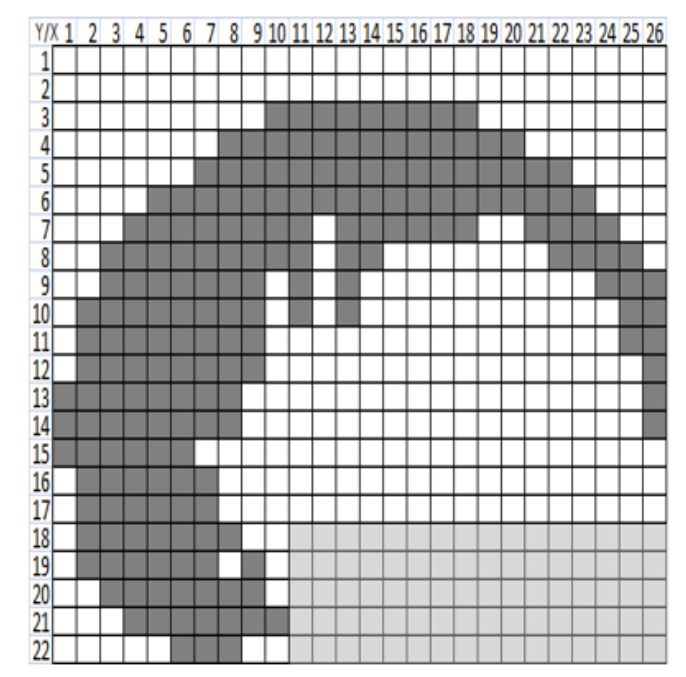

Fuente: elaboración propia

De esta forma se obtuvo la combinación de ángulos necesarios para alcanzar cada una de las coordenadas marcadas con gris oscuro en la

\section{Resultados}

El triple controlador se implementó en hojas de cálculo de Microsoft Excel ${ }^{\circledR}$ y se enlazó con una interfaz realizada en la versión 6.0 de Visual Basic, para utilizar los resultados del razonamiento difuso y hacer mover el brazo mediante el puerto serial del PC. Todo esto se probó con la intención de alcanzar la coordenada $(7,10)$ a partir de cualquier otra posición. figura 7, y así los tres subcontroladores se encargan de posicionar el brazo completo.

El brazo alcanza dicha coordenada cuando los ángulos de los tres motores miden $\left\{70^{\circ}, 20^{\circ}, 40^{\circ}\right\}$ respectivamente, pues se definieron esos valores como ángulo deseado al respectivo subcontrolador y cada motor mostró el comportamiento detallado en la figura 8.

Figura 8. Comportamiento de los tres motores del brazo-robot

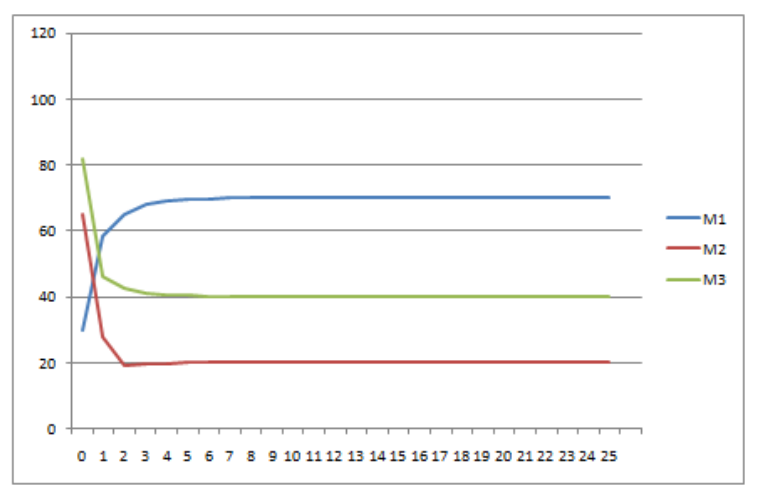

Fuente: elaboración propia 


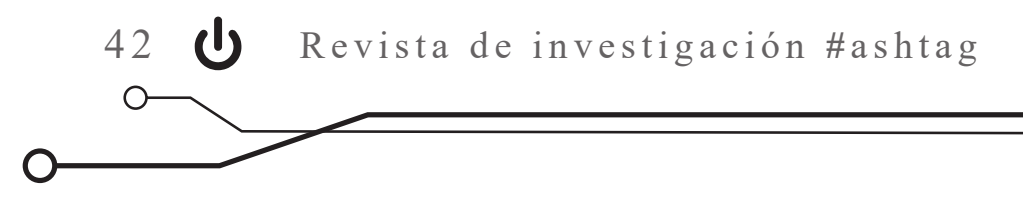

Esta gráfica muestra que los motores del brazo inician en $\left\{30^{\circ}, 65^{\circ}, 82^{\circ}\right\}$, y al cabo de 24 iteraciones terminan en $\left\{70^{\circ}, 20^{\circ}, 40^{\circ}\right\}$, con lo que se logra la posición deseada. En realidad no importa cuál haya sido la posición inicial, siempre

\section{Conclusiones}

En este artículo se presenta el diseño de un tiple controlador difuso tipo Mamdani, capaz de movilizar un brazo-robot constituido por tres servomotores, con el fin de hacerlo alcanzar cierta posición en un espacio 2D. Así puede alcanzar automáticamente secuencias ilimitadas de coordenadas, con el fin de recoger o entregar piezas y cuando se definan los valores $\left\{70^{\circ}, 20^{\circ}, 40^{\circ}\right\}$ como ángulos deseados, el brazo se posiciona en $(7,10)$, y a partir de ésta podría reposicionarse el brazo a una nueva coordenada, cuantas veces sea necesario.

en algún proceso automatizado. Sin embargo, en este artículo se asume que dichas secuencias son definidas por algún usuario, y en un trabajo futuro se puede indagar sobre cómo obtenerlas autónomamente, empleando un sistema de planificación artificial, adaptable a procedimientos variables.

\section{Referencias}

García, J., Medel, J., Sánchez, J. y Tequianez, A. (2009). Sistemas con lógica difusa. México: Instituto Politécnico Nacional.

Martín, B. y Sanz, A. (2006). Redes neuronales y sistemas borrosos. Madrid: Alfaomega.

Ponce, P. (2010). Inteligencia artificial con aplicaciones a la ingeniería. Madrid: Alfaomega.

Robayo, E. (2007). Control difuso: fundamentos y aplicaciones. Barranquilla: Editorial de la Universidad del Norte.

Sterpin, D. (2015). Apuntes de clase en la asignatura de control difuso [documento interno de trabajo]. Corporación Unificada Nacional de Educación Superior (CUN). 